\title{
One-year outcomes in schizophrenia after switching from typical antipsychotics to olanzapine in Japan: an observational study
}

This article was published in the following Dove Press journal:

Pragmatic and Observational Research

13 June 2012

Number of times this article has been viewed

\author{
Wenyu Ye' \\ Shinji Fujikoshi' \\ Naohiro Nakahara' \\ Michihiro Takahashi ${ }^{1,2}$ \\ Haya Ascher-Svanum ${ }^{3}$ \\ Tetsuro Ohmori ${ }^{4}$ \\ 'Eli Lilly Japan KK, Kobe, Japan; \\ ${ }^{2}$ Terauchi-Takahashi Psychiatric Clinic, \\ Ashiya, Japan; ${ }^{3}$ Lilly Corporate Center, \\ Eli Lilly and Company, Indianapolis, \\ IN, USA; ${ }^{4}$ Department of Psychiatry, \\ Tokushima University Hospital, \\ Tokushima, Japan
}

Correspondence: Naohiro Nakahara Eli Lilly Japan KK, Sannomiya Plaza Building, 7-I-5 Isogami-dori, Chuo-ku, Kobe 65I-0086, Japan

Tel +81782429054

Fax +81782429526

Email nakahara_naohiro@lilly.com
Background: The purpose of this study was to assess the 1-year clinical, functional, and safety-related outcomes following a switch to olanzapine of at least one typical antipsychotic drug in the previous regimen in the treatment of patients of schizophrenia in Japan.

Methods: Using data from a large 1-year prospective, multicenter, naturalistic study of olanzapine for the treatment of schizophrenia in Japan, patients who were switched from any oral typical antipsychotic to olanzapine were identified. Mixed models for repeated measures, controlling for baseline demographics, were utilized to assess outcomes for clinical and functional measures.

Results: Of the 262 patients who switched from typical antipsychotics to olanzapine, $41 \%$ were outpatients and $59 \%$ were inpatients. Most of these patients were switched due to poor medication efficacy $(71.0 \%)$ or medication intolerability $(25.6 \%)$. Most patients $(71.4 \%)$ completed the 1 -year study. Clinically and statistically significant $(P<0.01)$ improvements were observed in patient illness severity and health-related quality of life, including improvements in global symptom severity and in positive, negative, depressive, and cognitive symptoms. Over half of the patients $(58.3 \%)$ demonstrated a treatment response to olanzapine and $47.4 \%$ achieved symptom remission. Mean weight gain from baseline to endpoint was $2.31 \pm 4.72 \mathrm{~kg}$, with $30.4 \%$ of patients experiencing clinically significant weight gain (at least $7 \%$ of baseline weight).

Conclusion: During this 1-year naturalistic treatment of schizophrenia patients in Japan, switching from typical antipsychotics to olanzapine resulted in significant improvements in patients' clinical and functional outcomes. Approximately one-third of patients had clinically significant weight gain. These findings highlight the favorable benefit to risk profile of switching to olanzapine following failure on typical antipsychotics.

Keywords: olanzapine, treatment outcomes, schizophrenia

\section{Introduction}

Antipsychotic medications represent the cornerstone of treatment for schizophrenia and are effective for both reducing acute symptoms and for preventing future relapses..$^{1-5}$ In usual care settings, antipsychotic treatment is often a dynamic process that involves changing medications, ${ }^{6,7}$ primarily due to problems with medication efficacy, ${ }^{6,8}$ but also due to patient preferences, medication intolerability, and nonadherence.

In Japan, typical antipsychotics continue to be used frequently for the treatment of schizophrenia. ${ }^{9-11}$ However, the use of atypical antipsychotics (eg, risperidone, olanzapine, quetiapine, perospirone, and aripiprazole) is rapidly increasing. ${ }^{11}$ Olanzapine has been reported to be the second most frequently used atypical antipsychotic in Japan ${ }^{11}$ and may be a likely choice of medication following failure of a typical antipsychotic for patients with schizophrenia. 
Japan has been reported to have the highest level of psychiatric inpatient beds per capita. ${ }^{12}$ Many patients who are treated in hospitals could possibly be treated as outpatients in the community, ${ }^{12}$ but community-based psychiatric services to handle this load of patients are not fully developed in Japan. ${ }^{11}$ Understanding any differences in outcomes for patients with schizophrenia treated in inpatient versus outpatient settings may be of particular importance in Japan.

To understand better the outcomes for patients with schizophrenia who switch from a typical antipsychotic to olanzapine in usual care in Japan, we analyzed data for a subset of patients from a larger observational study. ${ }^{13}$ The objectives of this analysis were two-fold, ie, to assess clinical, functional, and safety-related outcomes following a switch from a typical antipsychotic to olanzapine in a 1-year naturalistic study of schizophrenia patients in Japan, and to compare treatment outcomes between inpatients and outpatients who were switched from a typical antipsychotic to olanzapine.

\section{Methods}

\section{Data source}

This post hoc analysis used data from a large $(n=1949)$ multicenter, naturalistic, 1-year, postmarketing surveillance study in Japan. ${ }^{13}$ Postmarketing surveillance studies are single-arm studies designed to identify occurrences of serious adverse reactions quickly and are required by the regulatory bodies in Japan as part of the approval process to market a new medication. Primary eligibility for the olanzapine postmarketing surveillance study included a diagnosis of schizophrenia based on Diagnostic and Statistical Manual of the American Psychiatric Association, Fourth Edition (DSM-IV) $)^{14}$ criteria and initiation of treatment with olanzapine. In this naturalistic, observational, and noninterventional study, all treatment decisions were left to the discretion of the treating clinician. The initiation of olanzapine could have been a patient's first antipsychotic treatment, a switch from another antipsychotic treatment, or augmentation of their current antipsychotic regimen. When patients discontinued olanzapine, they discontinued participation in the study. Study enrollment ran from November 2003 until July 2004. Data were collected at the baseline, 3-month, 6-month, and 12-month visits.

All study procedures were approved by the internal review boards at each of the participating medical facilities. Informed consent was obtained based on the rules at each participating institution.
Almost all of the 1949 patients screened in the parent study met all eligibility criteria (94.9\%, 1850 of 1949 patients). ${ }^{13}$ Most of the eligible patients (67.1\%, 1241 of 1850 patients) were not switched from an antipsychotic to olanzapine, but either had olanzapine added to their current antipsychotic regimen or were initiating a new course of antipsychotic treatment with olanzapine. The current analysis was further restricted to the 262 patients who were switched from an oral typical antipsychotic to olanzapine.

\section{Measures}

In order to avoid interference with usual-care processes, the procedures in this observational study were designed to capture only a limited amount of information. Invasive or cumbersome measures were not included.

The Clinical Global Impression-Schizophrenia (CGI-SCH) is a clinician-rated measure consisting of five ratings, ie, global severity, positive, negative, cognitive, and depressive symptoms. All ratings are made on an anchored scale ranging from no symptoms (0) to severe symptoms $(6) \cdot{ }^{15}$ The concurrent validity of the CGI-SCH subscales with the corresponding subscales from the more rigorous Positive and Negative Syndrome Scale ${ }^{16}$ has been found to range from 0.61 for depressive symptoms to 0.86 for positive symptoms, with the remaining correlation coefficients ranging from 0.75 to 0.80 . Interrater reliability has also been found to be moderately high (interclass correlation coefficients ranging from 0.73 to 0.82 ) for all but the depressive subscale $(0.64) .^{15}$

The European Quality of Life-5 Dimensions (EQ-5D) is a generic measure of health-related quality of life that includes a visual analog scale of overall health (ranging from 0 to 100) and five measures of specific dimensions (level of movement, control of environment, normal activities, pain/discomfort, and anxiety/depression). The ratings on the five dimensions are used to create health states that have been assigned values (utilities) ranging from death (0) to perfect health (1). ${ }^{17}$ The construct validity of the EQ-5D has been evaluated in a sample of individuals with schizophrenia: utility scores were moderately correlated with the PANSS Total Score $(-0.51)$ and subscales $(-0.20$ to -0.59$)$, and World Health Organization Quality of Life-Brief Questionnaire Overall Score $(0.55)$ and subscales $(0.32$ to 0.64$) .{ }^{18}$

The study also collected a broad array of information on treatment, functioning, and adverse events. Information on concomitant medications included drug name, dose, route of administration, start and stop dates, and therapeutic category (antipsychotic, anticholinergic, antidepressant, anxiolytic/ hypnotic, mood stabilizer, or other). In addition to the 
EQ-5D, measures of functioning included employment status (including working for pay) and number of social activities in the past 4 weeks $(0,1,2,3,4$, or $5+)$. The study included specific ratings of the following adverse events: dystonia/ akathisia/parkinsonism, tardive dyskinesia, decreased libido, amenorrhea/other menstrual dysfunction, gynecomastia, galactorrhea, and erectile/sexual dysfunction. The presence of any of the following medical complications was assessed at baseline: hypertension, hyperlipidemia, hepatic dysfunction, renal dysfunction, or other. Finally, body weight in kilograms was measured at each visit.

\section{Procedures}

This analysis was restricted to individuals who had been treated with typical antipsychotics prior to initiating olanzapine at baseline $(n=262)$. The treatment regimens for all patients included in this analysis had at least one typical antipsychotic replaced with olanzapine. Patients were classified as either inpatients or outpatients based on their treatment setting at baseline.

Symptomatic response and remission were defined using previously published definitions based on the CGI-SCH. Response was defined as an improvement of 2 points on the CGI-SCH global severity rating at any visit when the baseline rating was between 4 and 6 points, or a 1-point improvement at any visit when the baseline rating was between 1 and 3. ${ }^{19}$ Symptomatic remission was defined as mild symptoms (a score $\leq 2$ ) on the CGI-SCH positive, negative, cognitive, and global severity scores. ${ }^{20}$

Patients' body mass indices were categorized as follows: underweight $(<18.5)$, normal $(\geq 18.5, \leq 23)$, overweight $(>23, \leq 30)$, and obese $(>30)$. This categorization was based on the World Health Organization recommendations for Asians. ${ }^{21}$

Time to all-cause discontinuation has been described as a measure of effectiveness that incorporates both efficacy and tolerability. ${ }^{22}$ We defined time to all-cause discontinuation as the number of days between the date a patient initiated treatment with olanzapine and the date on which the patient discontinued taking olanzapine.

\section{Statistical methods}

At baseline, comparisons between the inpatient and outpatient groups were completed with $t$-tests for continuous variables and Chi-square tests for categorical variables. Changes over time for continuous outcome variables were assessed using mixed models for repeated measures with baseline covariates for age, sex, duration of illness, and presence of any medical complication. Changes in categorical outcome variables between baseline and postbaseline visits were assessed using McNemar's test with missing observations imputed. Time to all-cause discontinuation was assessed using survival analysis with log-rank tests. The survival curves were constructed using unadjusted Kaplan-Meier estimates. A sensitivity analysis was completed through use of the primary symptoms measure (CGI-SCH) using the subset of participants who were treated with olanzapine monotherapy throughout the study. This sensitivity analysis allowed for confirmation that the results were not due to use of concomitant antipsychotic medications. SAS software (version 9.1.3; SAS Institute Inc, Cary, NC) was used for all analyses. The level of significance was set at $P<0.05$.

\section{Results \\ Baseline characteristics}

The patients had been diagnosed with schizophrenia for an average duration of 19.5 years, were on average 46.9 years of age, and $51.9 \%$ were male. The inpatients (59.1\%) were older, with a longer history of schizophrenia, and greater severity of symptoms overall than the outpatients (see Table 1). The most common reason for discontinuing the previous typical antipsychotic was insufficient efficacy $(71.0 \%$ of patients) followed by medication intolerability $(25.6 \%)$.

This naturalistic study followed the switching process from at least one typical antipsychotic to olanzapine as instituted by the treating physicians. Olanzapine was initiated immediately after discontinuing the typical antipsychotic(s) for the majority of patients (69.5\%). The typical antipsychotic(s) and olanzapine overlapped for nine patients (3.4\%). The duration of overlap was less than 15 days for five patients, with the remaining patients having overlap durations of 17, 28, 56, and 83 days. Finally, there was a gap between discontinuation of the typical antipsychotic(s) and the initiation of olanzapine for $27.1 \%$ of patients. The gap was less than 7 days in length for all but six patients. Only $53.8 \%$ of patients were initiated on olanzapine monotherapy because some patients continued taking other antipsychotics.

\section{Treatment patterns}

Most patients completed the 1-year study (71.4\%), with $88.2 \%$ completing the 3 -month visit and $81.7 \%$ completing the 6-month visit. Discontinuation rates did not vary significantly between inpatients and outpatients. During the study, the average daily dose for olanzapine was $12.0 \pm 6.0 \mathrm{mg}$, including $9.9 \pm 5.5 \mathrm{mg}$ for outpatients and $13.4 \pm 5.9 \mathrm{mg}$ for inpatients $(P<0.001)$. Combination antipsychotic treatment 
Table I Comparison of baseline characteristics of outpatients and inpatients who switched from typical antipsychotic to olanzapine

\begin{tabular}{|c|c|c|c|c|}
\hline Characteristic & $\begin{array}{l}\text { Overall } \\
(n=262)\end{array}$ & $\begin{array}{l}\text { Outpatients } \\
(n=107)\end{array}$ & $\begin{array}{l}\text { Inpatients } \\
(\mathrm{n}=155)\end{array}$ & $\mathbf{P}$ \\
\hline Age (years), mean \pm SD & $46.9 \pm 14.6$ & $41.8 \pm 13.5$ & $50.5 \pm 14.3$ & $<0.001$ \\
\hline Male (\%) & 51.9 & 46.7 & 55.5 & 0.16 \\
\hline Duration of illness (years), mean $\pm \mathrm{SD}^{\dagger}$ & $19.5 \pm 14.4$ & $14.1 \pm 12.5$ & $23.3 \pm 14.4$ & $<0.001$ \\
\hline Any medical complications (\%) & 40.8 & 25.2 & 51.6 & $<0.001$ \\
\hline Working for pay $(\%)^{\ddagger}$ & 7.7 & 16.0 & 2.0 & $<0.001$ \\
\hline CGI-SCH global, mean $\pm \mathrm{SD}^{\ddagger}$ & $3.3 \pm 1.1$ & $3.0 \pm 1.0$ & $3.5 \pm 1.1$ & $<0.001$ \\
\hline $\mathrm{CGI}-\mathrm{SCH}$ positive, mean $\pm \mathrm{SD}^{\ddagger}$ & $2.7 \pm 1.5$ & $2.1 \pm 1.3$ & $3.1 \pm 1.5$ & $<0.001$ \\
\hline CGI-SCH negative, mean $\pm \mathrm{SD}^{\ddagger}$ & $3.1 \pm 1.3$ & $2.9 \pm 1.4$ & $3.2 \pm 1.3$ & 0.06 \\
\hline CGI-SCH cognitive, mean $\pm \mathrm{SD}^{\ddagger}$ & $2.9 \pm 1.3$ & $2.7 \pm 1.3$ & $3.1 \pm 1.4$ & 0.02 \\
\hline CGI-SCH depressive, mean $\pm \mathrm{SD}^{\ddagger}$ & $\mathrm{I} .7 \pm \mathrm{I} .4$ & $1.9 \pm 1.4$ & $1.5 \pm 1.4$ & 0.03 \\
\hline EQ-5D VAS, mean $\pm \mathrm{SD}^{\S}$ & $49.5 \pm 22.7$ & $46.6 \pm 21.0$ & $51.5 \pm 23.7$ & 0.10 \\
\hline $\mathrm{BMI}$, mean $\pm \mathrm{SD} \pi$ & $22.9 \pm 4.4$ & $23.4 \pm 4.0$ & $22.6 \pm 4.5$ & 0.15 \\
\hline BMI classification (\%) & & & & 0.01 \\
\hline Underweight $(<18.5)$ & 15.4 & 6.5 & 20.9 & \\
\hline Normal $(\geq 18.5, \leq 23)$ & 40.7 & 47.3 & 36.5 & \\
\hline Overweight $(>23, \leq 30)$ & 36.5 & 40.9 & 33.8 & \\
\hline Obese $(>30)$ & 7.5 & 5.4 & 8.8 & \\
\hline Reason for switch from typical (\%) & & & & 0.02 \\
\hline Insufficient efficacy & 71.0 & 70.1 & 71.6 & \\
\hline Medication intolerability & 25.6 & 20.6 & 29.0 & \\
\hline Patient request & 5.7 & 10.3 & 2.6 & \\
\hline Noncompliance & 3.8 & 4.7 & 3.2 & \\
\hline
\end{tabular}

Notes: $P$ values represent the contrast between inpatients and outpatients. ${ }^{t} n=207 ;{ }^{\ddagger} n=259 ;{ } n=257 ;{ }^{\pi} n=24$ I.

Abbreviations: SD, standard deviation; CGI-SCH, Clinical Global Impression-Schizophrenia; EQ-5D VAS, European Quality of Life-5 Dimensions visual analog scale; BMI, body mass index.

was common: less than half of the patients $(36.6 \%)$ were treated with olanzapine monotherapy throughout the study, including $41.1 \%$ of outpatients and $33.5 \%$ of inpatients $(P=0.21)$. The remaining $63.4 \%$ of patients were either treated with antipsychotic polypharmacy for at least one day or discontinued the study. Patients were less likely to be treated with concomitant oral atypical antipsychotics (26.7\%; inpatients $26.5 \%$, outpatients $27.1 \%$ ) than typical antipsychotics (49.2\%; inpatients $51.0 \%$, outpatients $46.7 \%$ ). The average chlorpromazine equivalent doses of concomitant antipsychotics were $264.8 \mathrm{mg} /$ day for inpatients and $166.4 \mathrm{mg} /$ day for outpatients at baseline and $203.6 \mathrm{mg}$ /day for inpatients and $199.0 \mathrm{mg} /$ day for outpatients at the end of the study. With the exception of antidepressants, the inpatients were significantly more likely to be treated with different classes of concomitant medications measured in the study (see Table 2).

\section{Effectiveness outcomes}

Most patients (71.4\%) continued olanzapine treatment for the full 1-year study period. There were no differences between inpatients and outpatients in time to all-cause discontinuation $(P=0.61$, see Figure 1$)$.
On all of the CGI-SCH subscales, patients improved after initiating treatment with olanzapine $(P<0.001$, see Figure 2). The differences between inpatients and outpatients were most pronounced for global severity, where the inpatients had statistically significant higher (ie, more symptomatic) scores at each visit $(P<0.05)$. On the positive subscale, inpatients had significantly higher scores at baseline, 3-month, and 6-month visits. On the cognitive subscale, inpatients had significantly higher scores at the 6-month and 12-month visits. On the positive, cognitive, and depressive subscales, there was a significant time by initial treatment setting interaction $(P<0.05)$ that appeared to reflect greater change for the outpatients between the 3-month and 6-month visits.

Table 2 Concomitant psychotropic medications at final study visit

\begin{tabular}{lllll}
\hline & $\begin{array}{l}\text { All } \\
\text { patients }\end{array}$ & Outpatients & Inpatients & P \\
\hline Anticholinergics & $37.8 \%$ & $29.3 \%$ & $43.2 \%$ & 0.03 \\
Antidepressants & $8.8 \%$ & $12.0 \%$ & $6.8 \%$ & 0.18 \\
Anxiolytics/hypnotics & $72.3 \%$ & $64.1 \%$ & $77.4 \%$ & 0.03 \\
Mood stabilizers & $18.9 \%$ & $10.9 \%$ & $24.0 \%$ & 0.01 \\
Other & $53.4 \%$ & $28.3 \%$ & $69.2 \%$ & $<0.001$ \\
\hline
\end{tabular}

Notes: $P$ values represent the contrast between inpatients and outpatients; the other category included both psychiatric and nonpsychiatric medications. 


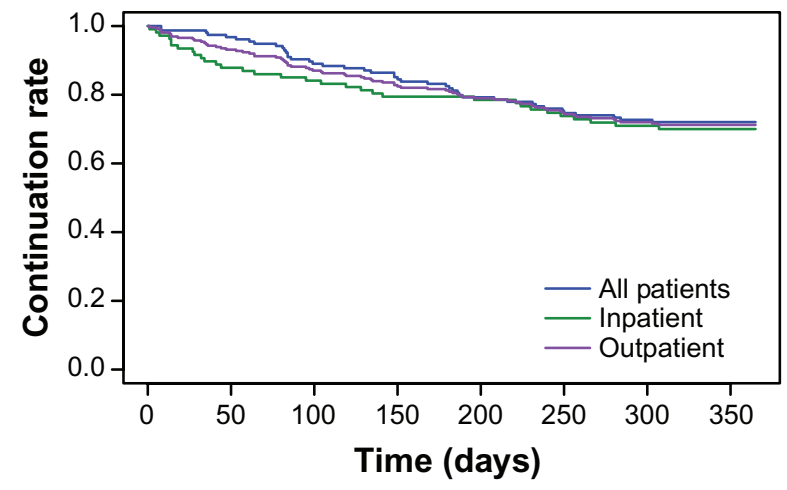

Figure I Time to all-cause discontinuation of olanzapine for all patients, inpatients, and outpatients.

Note: There was no significant difference in time to all-cause discontinuation between the inpatient and outpatient cohorts $(P=0.61)$.

The response rate was $58.3 \%$ and did not differ significantly between the inpatients $(56.8 \%)$ and the outpatients $(60.9 \%, P=0.53)$. However the remission rate $(47.4 \%)$ was significantly lower for the inpatients (39.3\%) than the outpatients $(61.3 \%, P=0.002)$.

Patient functioning improved after initiating treatment with olanzapine. On the EQ-5D visual analog scale, a broad rating of health-related quality of life, patients improved from a score of 50.3 at baseline to 66.9 at the 12-month visit $(P<0.001)$. There were no significant differences at any time point between inpatients and outpatients on this measure. Similarly, the utility score on the EQ-5D did not differ significantly between inpatients and outpatients at any point in time, but improved from 0.68 at baseline to 0.80 at the 12 -month visit for all patients $(P<0.001)$. Over the 1 -year study period, the percent of patients working for pay increased from $7.9 \%$ to $12.1 \%(P=0.01)$. The increase in percent of patients working for pay was higher for outpatients (increased from $17.4 \%$ to $25.0 \%, P=0.05$ ) than inpatients (increased from $2.0 \%$ to $4.5 \%, P=0.08$ ). The percent of patients engaging in five or more social activities in the previous 4 weeks increased from $17.1 \%$ at baseline to $24.6 \%$ at the 12-month visit $(P=0.004)$; for outpatients the change was from $26.1 \%$ to $39.1 \%(P=0.005)$, whereas for inpatients the change was from $11.5 \%$ to $15.6 \%(P=0.20)$.

\section{Tolerability outcomes}

Figure 3 displays the average observed body weight at each visit during the study. Patients who completed the 1-year study gained an average of $2.3 \mathrm{~kg}$ ( $2.4 \mathrm{~kg}$ for 64 outpatients and $2.2 \mathrm{~kg}$ for 100 inpatients). Overall, one in three patients (30.4\% of 227 patients) experienced clinically significant weight gain, defined as a $7 \%$ or greater increase over baseline during the study period. In terms of body mass index category,

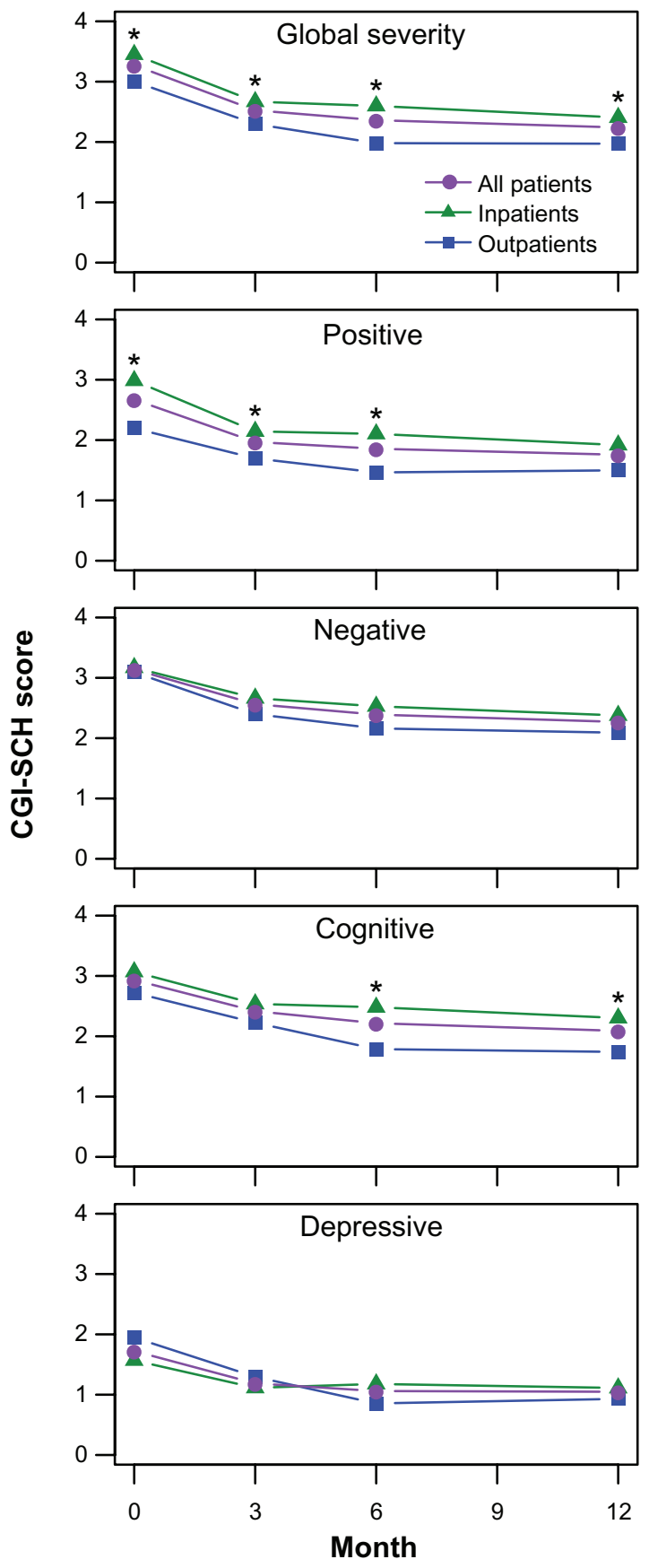

Figure 2 Change in CGI-SCH scores over the I-year study period. Notes: Each of the CGI-SCH subscale scores changed significantly from study entry to post-baseline visits $(P<0.00 \mathrm{I})$ for all patients, outpatients, and inpatients. *Significantly different at that visit $(P<0.05)$. Values represented the predicted means from mixed models for repeated-measures models that were adjusted for age, gender, duration of illness, and presence of any medical complication and may differ from the unadjusted baseline comparisons in Table I.

Abbreviation: CGI-SCH, Clinical Global Impression-Schizophrenia.

$2.8 \%$ of 213 patients decreased a category, $75.6 \%$ remained in the same category, and $21.6 \%$ increased a category. There were no reported cases of new onset diabetes.

For the adverse events measured in this study, new onset cases occurred in less than $5 \%$ of patients (see Table 3 ). 


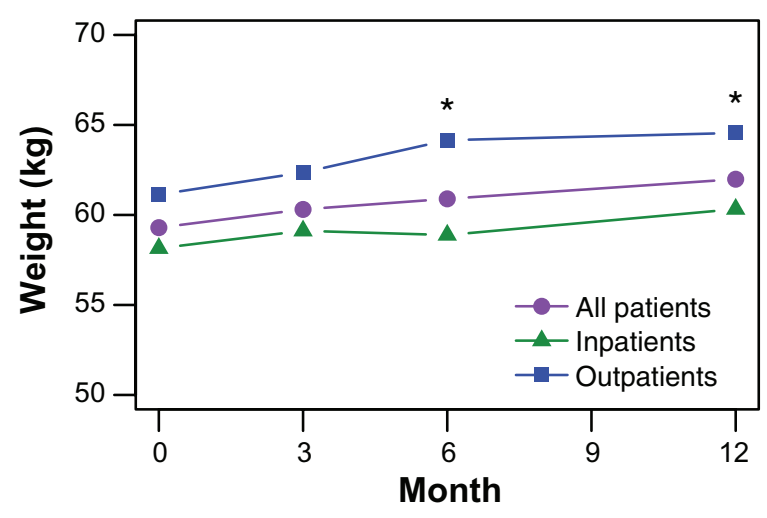

Figure 3 Observed body weight during the I-year study period. Note: *Average inpatient and outpatient body weight was significantly different at that visit $(P<0.05)$.

All five of the patients with new onset dystonia/akathisia/ parkinsonism and one of the two patients with new onset tardive dyskinesia were being treated concomitantly with typical antipsychotics.

\section{Sensitivity analysis}

In order to confirm that the results were not due to use of concomitant antipsychotics, analysis of the CGI-SCH scales was repeated using only participants treated with olanzapine monotherapy throughout the study. The monotherapy subsample consisted of 96 patients, including 44 outpatients and 52 inpatients. In this sensitivity analysis, significant changes from baseline to each post-baseline assessment for inpatients and outpatients on the CGI-SCH global severity and each of the subscales (negative, positive, depressive, and cognitive symptoms) were confirmed $(P<0.01)$. In addition, differences between inpatients and outpatients on global severity at 6 months, positive symptoms at 3 and 6 months, and cognitive symptoms at 6 and 12 months were confirmed. However, differences between inpatients and outpatients for

Table 3 New onset of adverse events

\begin{tabular}{lllll}
\hline Adverse events & $\begin{array}{l}\text { All } \\
\text { patients }\end{array}$ & Outpatients & Inpatients & $\boldsymbol{P}$ \\
\hline $\begin{array}{l}\text { Dystonia/akathisia/ } \\
\text { parkinsonism }\end{array}$ & $5(1.9 \%)$ & $\mathrm{I}(0.9 \%)$ & $4(2.6 \%)$ & 0.37 \\
$\begin{array}{l}\text { Tardive dyskinesia } \\
\text { Decreased libido }\end{array}$ & $2(0.8 \%)$ & $0(0.0 \%)$ & $2(1.3 \%)$ & 0.24 \\
$\begin{array}{l}\text { Amenorrhea/ } \\
\text { menstrual disturbance }\end{array}$ & $3(3.1 \%)$ & $2(2.1 \%)$ & $2(1.4 \%)$ & 0.70 \\
$\begin{array}{l}\text { Gynecomastia } \\
\text { Erectile/sexual }\end{array}$ & I (0.8\%) & I (2.1\%) & $0(0.0 \%)$ & 0.18 \\
dysfunction & $3(1.3 \%)$ & $2(2.2 \%)$ & I (0.8\%) & 0.38 \\
\hline
\end{tabular}

Notes: $P$ values represent the contrast between inpatients and outpatients; there were no cases of new-onset lactorrhea. global severity at baseline, 3 months, and 12 months, and on positive symptoms at baseline and 12 months were no longer significant in this subsample of patients treated only with olanzapine. The results of the sensitivity analysis confirmed significant improvements on all of the subscales of the primary measure of symptoms, but not all of the differences between inpatients and outpatients.

\section{Discussion}

Among Japanese inpatients and outpatients with schizophrenia who were treated with medication including typical antipsychotic drugs and who had at least one typical antipsychotic drug switched to olanzapine, the resulting treatment regimen was successful in most cases. Time to all-cause discontinuation can be thought of as a measure of overall effectiveness, incorporating efficacy, safety, and tolerability, ${ }^{22}$ and most (71.4\%) patients continued treatment with olanzapine for the full 1-year study period. In addition, patients showed significant improvements in symptom severity as measured by the global severity, positive, negative, cognitive, and depressive subscales of the CGI-SCH. Patient quality of life and level of functioning also improved, based on improvements in the EQ-5D measures, the percent of patients working for pay, and the percent of patients engaging in social activities. However, approximately one in three patients experienced clinically significant weight gain.

The inpatients appeared to be a unique subgroup of patients. Although both the inpatients and outpatients improved significantly over the study period, the inpatients had a different pattern of response in positive, cognitive, and depressive symptoms that was marked by less improvement than in the outpatients between the 3-month and 6-month visits. In addition to greater symptom response, the outpatients had significantly greater average weight at the 6-month and 12-month visits, which is consistent with past research showing a link between greater treatment response and greater weight gain. ${ }^{23,24}$ The inpatients were also treated with higher doses, were more likely to be treated with combination antipsychotic therapy, had more severe symptoms throughout the study, were less likely to achieve symptomatic remission, and had lower rates of participation in social activities. Our findings replicate previous research showing that inpatients have more severe symptoms, ${ }^{25,26}$ tend to be treated with higher doses of antipsychotics, ${ }^{25}$ and have greater needs for care. ${ }^{26}$

The results found in this study are similar to those of other typical to atypical switch studies in Japan. 
One study followed patients who switched from typical antipsychotics to the atypical antipsychotic risperidone and reported significant reductions in symptoms of schizophrenia and significant reductions in the use of anticholinergic medications. ${ }^{27}$ Another Japanese study followed male patients with schizophrenia who were switched to an atypical antipsychotic (olanzapine, quetiapine, or perospirone) and reported significant improvements in symptoms of schizophrenia, health-related quality of life, reduced use of anticholinergic drugs, and reductions in elevated prolactin levels. ${ }^{28}$ However, little published research has documented the outcomes in Japan following a switch from typical antipsychotics specifically to olanzapine.

In Europe, a large observational study compared patients who were switched to olanzapine from a variety of antipsychotics (either typical or atypical) with those who were switched away from olanzapine. Patients switched to olanzapine were significantly more likely to respond to treatment, significantly less likely to report extrapyramidal symptoms, and significantly less likely to report loss of libido than patients switched away from olanzapine. ${ }^{29}$ A large observational study in schizophrenia, that was relatively similar to the current study, followed Asian patients from China, the Philippines, South Korea, and Taiwan who were switched from typical antipsychotics to olanzapine. This similar study found significant improvements in symptoms of schizophrenia, health-related quality of life, and involuntary movements, and a greater weight gain. ${ }^{30}$ The findings from the current study, which specifically documents the naturalistic outcomes of patients with schizophrenia in the Japanese health care system who were switched from typical antipsychotics to olanzapine are consistent with the findings from studies in other geographic regions.

Although not directly comparable with the switching methodology used in the current study, the findings are also consistent with several head-to-head studies. In naturalistic, noninterventional, observational studies across a variety of geographies, relative to patients with schizophrenia treated with typical antipsychotics, olanzapine-treated patients have remained on treatment longer, ${ }^{31}$ had greater reductions in CGI-SCH global severity rating, ${ }^{19}$ greater response rates, ${ }^{31}$ greater improvements in quality of life, ${ }^{6,32}$ greater odds of engaging in social activities, ${ }^{19}$ lower rates of tardive dyskinesia, ${ }^{31}$ fewer extrapyramidal symptoms, ${ }^{31,32}$ fewer adverse events related to sexual functioning, ${ }^{31}$ and greater weight gain. ${ }^{32}$ In randomized controlled trials, a recent meta-analysis reported that relative to treatment with typical antipsychotics, olanzapine was associated with greater reductions in overall, positive, negative, and depressive symptoms, fewer extrapyramidal symptoms, and greater weight gain. ${ }^{33}$ Unlike head-to-head randomized clinical trials, when patients fail a given medication in usual care, the next treatment is not chosen at random and appears to reflect the issues resulting in the previous medication's discontinuation. ${ }^{34}$ The switching methodology more closely reflects the clinical challenge of finding the right medication for each individual patient. Our results highlight the importance of finding the right medication for the right patient at a given point in time.

\section{Limitations}

This observational study was designed to capture treatment outcomes for Japanese patients with schizophrenia in usual clinical care. The primary focus of the olanzapine postmarketing surveillance study was to identify potential safety issues after olanzapine was introduced in Japan. Design considerations favoring external validity were given precedence over those favoring internal validity. Consistent with usual clinical care, treatment was not blinded; therefore, patient and physician expectations may have affected outcomes. The centers that agreed to participate in this olanzapine treatment study may not have been fully representative of all treatment centers in Japan. Because there was no control group in this single-arm study, we cannot be certain that the improvement was due to treatment with olanzapine rather than simply an artifact of time. The results of this study do not provide information about the relative effectiveness of olanzapine versus other antipsychotics, only information about outcomes of olanzapine following a switch from a typical antipsychotic. Finally, antipsychotic polypharmacy was common in this study, which is consistent with other studies of usual care in Japan. ${ }^{9-11}$ Although the sensitivity analyses using only patients treated with olanzapine monotherapy confirmed the improvements, we cannot be certain that the improvements were not due to other nonantipsychotic medications or psychosocial treatments.

\section{Conclusion}

In this 1-year naturalistic study of patients with schizophrenia in Japan, inpatients and outpatients who were switched from typical antipsychotics to olanzapine experienced clinically and statistically significant improvements in their clinical and functional outcomes. One-third of patients had a clinically significant weight gain. Current findings highlight the 
favorable benefit to risk profile of switching to olanzapine therapy following treatment failure on typical antipsychotics among Japanese patients with schizophrenia.

\section{Disclosure}

Technical writing support was provided by Michael Stensland of Agile Outcomes Research Inc, Rochester, MN, and Susan Dennett of Strategic Health Outcomes Inc, Carmel, IN. WY, SF, and NN are full-time employees of Eli Lilly Japan. MT is a contract employee for Eli Lilly Japan. HA-S is a full-time employee of Eli Lilly and Company. WY, SF, NN, MT, and HA-S are all minor stockholders in Eli Lilly and Company. This funded research at the request of Eli Lilly Japan was undertaken under the agreement of the funded research condition of publishing to Article 4 clause 1 of the University of Tokushima School of Medicine.

\section{References}

1. Buchanan RW, Kreyenbuhl J, Kelly DL, et al. The 2009 schizophrenia PORT psychopharmacological treatment recommendations and summary statements. Schizophr Bull. 2010;36(1):71-93.

2. Canadian Psychiatric Association. Clinical practice guidelines. Treatment of schizophrenia. Can J Psychiatry. 2005;50(13 Suppl 1): $7 \mathrm{~S}-57 \mathrm{~S}$.

3. Falkai P, Wobrock T, Lieberman J, Glenthoj B, Gattaz WF, Möller HJ. World Federation of Societies of Biological Psychiatry (WFSBP) guidelines for biological treatment of schizophrenia. Part 1: acute treatment of schizophrenia. World J Biol Psychiatry. 2005;6(3): 132-191.

4. Falkai P, Wobrock T, Lieberman J, Glenthoj B, Gattaz WF, Möller HJ. World Federation of Societies of Biological Psychiatry (WFSBP) guidelines for biological treatment of schizophrenia. Part 2: longterm treatment of schizophrenia. World J Biol Psychiatry. 2006;7(1): $5-40$.

5. Lehman AF, Lieberman JA, Dixon LB, et al. Practice guideline for the treatment of patients with schizophrenia, second edition. Am J Psychiatry. 2004;161(Suppl 2):1-56.

6. Lieberman JA, Stroup TS, McEvoy JP, et al. Effectiveness of antipsychotic drugs in patients with chronic schizophrenia. $N$ Engl J Med. 2005;353(12):1209-1223.

7. Park S, Ross-Degnan D, Adams AS, Sabin J, Kanavos P, Soumerai SB. Effect of switching antipsychotics on antiparkinsonian medication use in schizophrenia: population-based study. Br J Psychiatry. 2005;187: $137-142$.

8. Liu-Seifert H, Adams DH, Kinon BJ. Discontinuation of treatment of schizophrenic patients is driven by poor symptom response: a pooled post-hoc analysis of four atypical antipsychotic drugs. BMC Med. 2005;3: 21-30.

9. Bitter I, Chou JC-Y, Ungvari GS, et al. Prescribing for inpatients with schizophrenia: an international multi-center comparative study. Pharmacopsychiatry. 2003;36(4):143-149.

10. Chong MY, Tan CH, Fujii S, et al. Antipsychotic drug prescription for schizophrenia in East Asia: rationale for change. Psychiatry and Clin Neurosci. 2004;58(1):61-67.

11. Shinfuku N, Tan C-H. Pharmacotherapy for schizophrenic inpatients in East Asia - changes and challenges. Int Rev Psychiatry. 2008;20(5): 460-468.

12. Oshima I, Mino Y, Inomata Y. How many long-stay schizophrenia patients can be discharged in Japan? Psychiatry Clin Neurosci. 2007; 61(1):71-77.
13. Kuramochi M, Ono H, Nakahara N, et al. Health outcome survey of olanzapine therapy in patients with schizophrenia: Analytical results of the post-marketing special survey of olanzapine. Jpn J Clin Psychopharmacol. 2009;12(1):71-89.

14. American Psychiatric Association. Diagnostic and Statistical Manual of Mental Disorders DSM-IV. 4th ed. Washington, DC: American Psychiatric Association; 1994.

15. Haro JM, Kamath SA, Ochoa S, et al. The Clinical Global ImpressionSchizophrenia scale: a simple instrument to measure the diversity of symptoms present in schizophrenia. Acta Psychiatr Scand Suppl. 2003; 416:16-23.

16. Kay SR, Opler LA, Fiszbein A. Positive and Negative Syndrome Scale (PANSS) User's Manual. North Tonawanda, NY: Multi-Health Systems Inc; 2000.

17. The EuroQol Group. EuroQol - a new facility for the measurement of health-related quality of life. Health Policy. 1990;16(3): 199-208.

18. Prieto L, Sacristán JA, Hormaechea JA, Casado A, Badia X, Gómez JC. Psychometric validation of a generic health-related quality of life measure (EQ-5D) in a sample of schizophrenic patients. Curr Med Res Opin. 2004;20(6):827-835.

19. Haro JM, Edgell ET, Novick D, et al. Effectiveness of antipsychotic treatment for schizophrenia: 6-month results of the Pan-European Schizophrenia Outpatient Health Outcomes (SOHO) study. Acta Psychiatr Scand. 2005;111(3):220-231.

20. Haro JM, Novick D, Suarez D, Alonso J, Lépine JP, Ratcliffe M. Remission and relapse in the outpatient care of schizophrenia: three-year results from the Schizophrenia Outpatient Health Outcomes study. $J$ Clin Psychopharmacol. 2006;26(6):571-578.

21. WHO Expert Consultation. Appropriate body-mass index for Asian populations and its implications for policy and intervention strategies. Lancet. 2004;363(9403):157-163.

22. Stroup TS, McEvoy JP, Swartz MS, et al. The National Institute of Mental Health Clinical Antipsychotic Trials of Intervention Effectiveness (CATIE) project: schizophrenia trial design and protocol development. Schizophr Bull. 2003;29(1):15-31.

23. Ascher-Svanum H, Stensland M, Zhao Z, Kinon BJ. Acute weight gain, gender, and therapeutic response to antipsychotics in the treatment of patients with schizophrenia. BMC Psychiatry. 2005;5:3.

24. Ascher-Svanum H, Stensland MD, Kinon BJ, Tollefson GD. Weight gain as a prognostic indicator of therapeutic improvement during acute treatment of schizophrenia with placebo or active antipsychotic. J Psychopharmacol. 2005;19(Suppl 6):110-117.

25. Soni SD, Mallik A, Reed P, Gaskell K. Differences between chronic schizophrenic patients in the hospital and in the community. Hosp Community Psychiatry. 1992;43(12):1233-1238.

26. Nakanishi M, Setoya Y, Kodaka M, et al. Symptom dimensions and needs of care among patients with schizophrenia in hospital and the community. Psychiatry Clin Neurosci. 2007;61(5):495-501.

27. Nakanishi S, Kunugi H, Murray RM, Nojima S, Ogawa T, Takahashi T. Effects of switching from conventional antipsychotics to risperidone in Japanese patients with chronic schizophrenia. Psychiatry Clin Neurosci. 2006;60(6):751-757.

28. Kaneda Y, Kawamura I, Fujii A, Ohmori T. Impact of a switch from typical to atypical antipsychotic drugs on quality of life and gonadal hormones in male patients with schizophrenia. Neuro Endocrinol Lett. 2004;25(1-2):135-140.

29. Novick D, Haro JM, Suarez D, Marques-Teixeira J, Naber D. Clinical consequences of switching antipsychotic drugs in outpatients with schizophrenia: 36-month results from the European Schizophrenia Outpatient Health Outcomes study. Int Clin Psychopharmacol. 2008;23(4): 203-208.

30. Lu Z, Hu J, Chen CK, et al. Effectiveness and safety of olanzapine in the treatment of schizophrenia among Asian patients switching from conventional antipsychotics. Prog Neuropsychopharmacol Biol Psychiatry. 2007;31(1):32-40. 
31. Dossenbach M, Dyachkova Y, Pirildar S, et al. Effects of atypical and typical antipsychotic treatments on sexual function in patients with schizophrenia: 12-month results from the Intercontinental Schizophrenia Outpatient Health Outcomes (IC-SOHO) study. Eur Psychiatry. 2006;21(4):251-258.

32. Montes JM, Ciudad A, Gascón J, Gómez JC. Safety, effectiveness, and quality of life of olanzapine in first-episode schizophrenia: a naturalistic study. Prog Neuropsychopharmacol Biol Psychiatry. 2003;27(4): 667-674.
33. Leucht S, Corves C, Arbter D, Engel RR, Li C, Davis JM. Secondgeneration versus first-generation antipsychotic drugs for schizophrenia: a meta-analysis. Lancet. 2009;373(9657):31-41.

34. Stroup TS, Lieberman JA, McEvoy JP, et al. Results of phase 3 of the CATIE schizophrenia trial. Schizophr Res. 2009;107(1):1-12.

Pragmatic and Observational Research

\section{Publish your work in this journal}

Pragmatic and Observational Research is an international, peer-reviewed, open access journal that publishes data from studies designed to reflect more closely medical interventions in real-world clinical practice compared with classical randomized controlled trials (RCTs). The manuscript management system is completely online and includes a very quick and fair peer-review

Submit your manuscript here: http://www.dovepress.com/pragmatic-and-observational-research-journal

\section{Dovepress}

system. Visit http://www.dovepress.com/testimonials.php to read real quotes from published authors. 